\title{
Youth Aerobic Fitness
}

\author{
Neil Armstrong \\ University of Exeter
}

Aerobic (or cardiorespiratory or cardiopulmonary) fitness reflects the integrated ability to deliver oxygen from the atmosphere to the skeletal muscles and to utilize it to generate energy to support muscle activity during exercise. Aerobic fitness is the most researched physiological variable in the history of pediatric exercise science, but its assessment, interpretation, and relationship with current and future health remain topics of lively debate as evidenced by this Special Issue of Pediatric Exercise Science.

Maximal oxygen uptake ( $\left.\dot{\mathrm{V}}_{2} \mathrm{max}\right)$, the highest rate at which an individual can consume oxygen during exercise, limits the performance of aerobic exercise and is internationally recognized as the best single measure of adults' aerobic fitness. $\dot{\mathrm{V}}_{2} \max$ serves as a biomarker for the development and severity of various health outcomes, and a high $\dot{\mathrm{VO}}_{2} \max$ is a pre-requisite for elite performance in many sports. However, $\dot{\mathrm{V}}_{2}$ max does not define all aspects of aerobic fitness. Cardiopulmonary exercise tests (CPETs), which rely solely on $\mathrm{V}_{2}$ max, may mask clinical insights that can be gleaned from submaximal data and/or relationships between physiological variables. Similarly, in several sports and in everyday life, the ability to engage in rapid changes in exercise intensity is at least as important as $\mathrm{VO}_{2}$ max and best described by the transient kinetics of pulmonary $\dot{\mathrm{VO}}_{2}$.

The first scientist to attempt to measure $\dot{\mathrm{V}}_{2}$ during exercise was Antoine-Laurent Lavoisier in the 1770s, but it is the work of Archibald Vivian Hill in the 1920s, which is generally credited with introducing the concept of $\mathrm{VO}_{2}$ max to exercise science (30). By the late 1930s, data purporting to describe the $\dot{\mathrm{VO}}_{2}$ max of boys had been reported (41), and in 1952, Åstrand (10) published his seminal studies of the "physical work capacity" of both boys and girls. Åstrand noted that only 70 of the 140 schoolchildren he tested using a discontinuous, incremental exercise protocol to voluntary exhaustion satisfied the conventional $\mathrm{VO}_{2}$ plateau criterion for confirming achievement of $\dot{\mathrm{V}}_{2}$ max. This phenomenon was largely ignored for decades, and when it was addressed and the term peak $\dot{\mathrm{VO}}_{2}$ introduced, it was often rejected by scientific journals as being a reflection of failure to elicit maximal values of $\mathrm{VO}_{2}$ in children.

It is challenging to interpret aerobic fitness during childhood and adolescence as changes in growth and maturation governed by the running of individual biological clocks must be accounted for. Typically, pediatric exercise scientists and clinicians have ignored maturity status and addressed changes in body size by attempting to "control" for body mass. In the first laboratory investigation of boys' "physical fitness," Robinson (41), without a scientific rationale or statistical justification, presented his $\mathrm{VO}_{2}$ data in ratio with

Armstrong is with the Children's Health and Exercise Research Centre, University of Exeter, Exeter, United Kingdom. Armstrong (N.Armstrong@exeter.ac.uk) is corresponding author. body mass (ie, in $\mathrm{mL} \cdot \mathrm{kg}^{-1} \cdot \mathrm{min}^{-1}$ ) and initiated an approach for "controlling" the children's body mass that has continued to the present day. In 1949, Tanner (50) demonstrated the fallacy of 1:1 ratio scaling with body mass and showed how spurious relationships emerge when these data are correlated with other healthrelated variables. Subsequent studies using allometric analyses revealed how simply dividing peak $\mathrm{V}_{2}$ with body mass has not only confounded understanding of the development of aerobic fitness (eg, 6,9,58) but also misinterpreted its relationship with markers of youth health (eg, 8,31,36). Yet, the vast majority of published articles still report young people's peak $\mathrm{VO}_{2}$ in ratio with body mass (15), and the spurious use of these data in subsequent statistical analyses with other health-related variables persists (36).

In an Invited Symposium at the 1999 Conference of the American College of Sports Medicine (subsequently published as a Special Issue of Pediatric Exercise Science), it was argued that despite over 60 years of intensive investigation, the assessment and interpretation of youth aerobic fitness is shrouded in controversy (5). Twenty years later, the topic is still contentious; the vast majority of published data are cross-sectional and well-designed longitudinal studies are sparse. Peak $\mathrm{VO}_{2}$ is now recognized as the "gold-standard" measure of young people's aerobic fitness, but pediatric exercise scientists continue to wrestle with factors related to its rigorous determination. By contrast, there is a view that relying predominantly on maximal values in CPETs obfuscates clinically useful insights that can be obtained from submaximal data and innovative data analytics. Some researchers suggest that the responses of physiological variables at the onset and/or offset of an exercise challenge may be as (or more) important than peak effort. Others promote the estimation of peak $\mathrm{VO}_{2}$ from field test performance scores as a valid alternative to rigorous determination of peak $\dot{\mathrm{VO}}_{2}$. Some writers advocate the use of fixed values of peak $\dot{\mathrm{V}} \mathrm{O}_{2}$ in ratio with body mass to identify "clinical red flags" for 8- to 18 -year-olds. On the other hand, some scientists believe that to elucidate the development of aerobic fitness and its relationship with present and future health, it must be interpreted in relation to sex-specific, concurrent changes in a range of chronological ageand maturity status-driven morphological and physiological variables. These issues are addressed in the reviews, commentaries, letters, and original research papers presented in this timely Special Issue of Pediatric Exercise Science.

\section{Peak Oxygen Uptake}

In the opening review, Falk and Dotan (24) provide a perceptive analysis of the principal issues relating to the measurement of peak $\dot{\mathrm{V}}_{2}$. Methodological factors such as treadmill and cycle ergometry, exercise test protocols, and respiratory gas collection and analysis are critically examined. Potential pitfalls are identified, and the need for scientific rigor in the determination of peak $\mathrm{VO}_{2}$ is 
stressed. The distinction between $\dot{\mathrm{VO}}_{2} \max$ and peak $\dot{\mathrm{V}} \mathrm{O}_{2}$ is clarified, the widespread use of secondary criteria to confirm a maximal effort is questioned, and the efficacy of verification tests of peak $\mathrm{VO}_{2}$ is discussed. Falk and Dotan (24) cite the research of Barker et al (14) who proposed that to confirm a maximal value of $\dot{\mathrm{VO}}_{2}$, following completion of a ramp test to voluntary exhaustion, participants should undergo a verification test at an exercise intensity of $105 \%$ of the peak power output in the ramp test. In a research article in this Special Issue, Barker and colleagues (46) further develop their original work and conclude that the utility of a verification test to confirm that maximal values of $\dot{\mathrm{VO}}_{2}$ attained in a preceding ramp test is not affected by sex, body mass, or fitness status. They recommend that use of a verification test should replace secondary criteria (ie, predicted values of heart rate, respiratory exchange ratio, or blood lactate accumulation) in confirming maximal values of $\dot{\mathrm{V}} \mathrm{O}_{2}$. Interestingly, a proposal to adopt verification tests with adults (39) has recently stimulated a lively discussion elsewhere $(11,20,40)$.

Falk and Dotan (24) comment that although it makes little sense, numerous published reviews group together data from treadmills, cycle ergometers, and field test predictions of aerobic fitness to describe typical values of peak $\mathrm{VO}_{2}$. They reinforce the view that credible international "norms" for youth aerobic fitness are not available and emphasize that published norms of peak $\mathrm{V}_{2}$ should be treated with caution, as they do not accommodate maturity status and related body size differences that directly affect $\dot{\mathrm{VO}}_{2}$. The authors note that ratio scaling does not usually account for body mass and conclude that due to large changes during maturation properly accounting for body mass is essential.

Welsman and Armstrong (57) take up the challenge of interpreting cross-sectional peak $\mathrm{VO}_{2}$ data in relation to body mass. They demonstrate that although body mass can be appropriately controlled for using allometric scaling, the current practice of dividing young people's peak $\mathrm{VO}_{2}$ with body mass is not founded on a sound scientific or statistical rationale. They outline the history of the adoption and use of ratio scaling in pediatric exercise science and comment that they know of no other scientific discipline where a "convenient and traditional" but evidently fallacious statistical method is recognized by scientific journals as an acceptable alternative to rigorous statistical justification. They explain the statistical assumptions on which ratio scaling is based and draw on $\sim 1000$ peak $\dot{\mathrm{VO}}_{2}$ determinations from 20 of their published crosssectional studies to demonstrate empirically that the assumptions are rarely met in groups of young people. They emphasize that if the use of ratio scaling cannot be demonstrated to appropriately describe young people's aerobic fitness, then any comparisons, conclusions, or recommendations based on ratio-scaled peak $\dot{\mathrm{VO}}_{2}$ are likely to be spurious. They suggest that children and adolescents with levels of aerobic fitness which raise "clinical red flags" based on peak $\dot{\mathrm{VO}}_{2}$ divided by body mass should be investigated not for cardiovascular risk but for what Tanner referred to as "no more formidable a disease than statistical artefact" (50, p. 3).

In a Letter to the Editor in response to Welsman and Armstrong's Commentary, Blais et al (16) comment that they too, "have observed, with some disbelief, the ongoing and widespread utilization of ratio scaling with body mass, despite overwhelming scientific evidence of its many drawbacks." They outline their development of equations based on multivariate regression models that predict means and range of normality that are independent of sex and body size. Recognizing the critical importance of data interpretation in clinical practice, Blais et al (16) indicate that they are currently testing the prediction equations, derived from cycle ergometer tests with healthy youth, in children with congenital heart diseases and cardiomyopathies to assess their diagnostic and prognostic values. Welsman and Armstrong (56) welcome both the supportive comments and the exploration of new methodology, which they hope will encourage others to reconsider how they interpret youth aerobic fitness and avoid spurious correlations with other health-related variables. Welsman and Armstrong (56) reiterate how they have persistently demonstrated that even with crosssectional studies, there is a need to concurrently control for age, maturity status, and a range of morphological variables but argue that longitudinal studies are required to effectively interpret developmental changes in aerobic fitness. They refer readers to their recent multiplicative allometric modeling articles in this Special Issue and elsewhere.

In their research article focusing on longitudinal data, Armstrong and Welsman (7) further develop their Commentary on cross-sectional data and apply a multiplicative allometric modeling approach to 1057 determinations of 10- to 18-yearolds' peak $\mathrm{VO}_{2}$. The multilevel models enable the effects of age, body mass, fat-free mass, and maturity status to be partitioned concurrently within an allometric framework to provide a flexible and sensitive interpretation of the development of peak $\mathrm{V}_{2}$. The baseline model shows that body mass and body fatness controlled for peak $\mathrm{VO}_{2}$ is higher in boys than in girls and increases with age in both sexes with the age effect smaller in girls. Sex-specific models illustrate that the ratio-scaled peak $\dot{\mathrm{VO}}_{2}$ interpretation of age-related aerobic fitness is specious and expose the fallacy of using ratio-scaled data as "clinical red flags" or age-related norms. The data demonstrate that, in direct conflict with the ratio-scaled interpretation of aerobic fitness, with body mass controlled for both age and maturity status, they have positive effects on peak $\dot{\mathrm{VO}}_{2}$. Moreover, the modeled data show that it is maturity status-driven fat-free mass, and not body mass, which is the most powerful morphological influence on the development of peak $\dot{\mathrm{VO}}_{2}$.

\section{Field Performance Tests}

In his Editor's Notes in the first volume of Pediatric Exercise Science, Founding Editor Tom Rowland commented that "there is little in the field of pediatric exercise that has stimulated as much emotional debate as the components, interpretation, and values of mass physical fitness testing of children and youth" (42, p. 289). He initiated a dialog in Pediatric Exercise Science (see 38,48), which is rekindled in the present issue by Jo Welsman (55). In her Commentary, she notes that articles predicting aerobic fitness from field performance scores have percolated through Pediatric Exercise Science for 30 years and argues that it really is time to move on from mass performance testing and focus on scientific rigor, or as Rowland (43) pithily commented in 1995, "The horse is dead. Let's dismount." By contrast, Tomkinson et al (53) have, through the assembly of large international data sets, stimulated a resurgence of interest in field performance tests, in particular the 20 -m shuttle run test $(20 \mathrm{mSRT})$. Despite a recent meta-analysis revealing that over half of published correlation coefficients between 20mSRT performance scores and children's peak $\mathrm{VO}_{2}$ explain less than $50 \%$ of the variance in peak $\mathrm{VO}_{2}$ (33), the last 2 years have witnessed a plethora of publications using variants of the 20mSRT to estimate/predict the ratio-scaled peak $\mathrm{VO}_{2}$ of children and to relate their $20 \mathrm{mSRT}$ score to current and future health $(2,23)$.

In a wide-ranging review, Tomkinson et al (53) acknowledge "gas-analyzed peak $\mathrm{VO}_{2}$ " as the criterion measure of aerobic 
fitness and note that with children, the 20mSRT has "moderate" criterion validity against peak $\dot{\mathrm{VO}}_{2}$. They calculate that the $95 \%$ range for a true peak $\mathrm{VO}_{2}$ value estimated from $20 \mathrm{mSRT}$ performance is $\sim 24 \%$, but contend that field testing offers a simple, cheap, practical alternative to gas analysis. They develop a case for the advantages offered by the $20 \mathrm{mSRT}$ over other field performance tests and outline the measurement variability, validity, reliability, and feasibility of $20 \mathrm{mSRT}$ performance scores. The authors promote the interpretation of $20 \mathrm{mSRT}$ predictions of peak $\dot{\mathrm{VO}}_{2}$ ratio scaled with body mass with reference to health-related criterion-referenced standards and normative-referenced standards. They state that the best available health-related international standards are the "clinical red flags to avoid cardiovascular disease risk" proposed by Ruiz et al (44). These "clinical red flags" classify prepubertal, pubertal, and postpubertal (8- to 18-y-olds) on the basis of a single sex-specific "cut point" and specify that values of peak $\dot{\mathrm{VO}}_{2}$ below 42 and $35 \mathrm{~mL} \cdot \mathrm{kg}^{-1} \cdot \mathrm{min}^{-1}$ should raise concern among boys and girls, respectively. As Tomkinson et al (53) report that the typical international norm value of 9- to 17-yearolds' peak $\mathrm{VO}_{2}$ estimated from $20 \mathrm{mSRT}$ performance scores is $40 \mathrm{~mL} \cdot \mathrm{kg}^{-1} \cdot \mathrm{min}^{-1}$, the review paints a depressing picture of international pediatric health. Published studies offering normative international and national reference standards for peak $\mathrm{V}_{2}$ predicted from $20 \mathrm{mSRT}$ performance are comprehensively tabulated, and the authors advise using standardized test scores to facilitate international and national comparisons of norms for healthy children and youth. Tomkinson et al (53) conclude by recommending that $20 \mathrm{mSRT}$ performance scores be considered as an international population health surveillance measure to help better understand the impact of health-promoting policies and interventions.

As discussed by Tomkinson et al (53), there are several variants of 20mSRT protocols and prediction equations with the Progressive Aerobic Cardiovascular Endurance Run (PACER) test-one of the most popular, particularly in North America. Scott et al (47) describe the development and validation of a prediction equation to estimate peak $\dot{\mathrm{VO}}_{2}$ in the PACER test. Unlike earlier attempts to predict peak $\dot{\mathrm{VO}}_{2}$ that have generally used treadmill-determined peak $\dot{\mathrm{VO}}_{2}$ as the criterion measure of aerobic fitness, the authors recorded $\mathrm{VO}_{2}$ during PACER tests. Peak $\dot{\mathrm{VO}}_{2}$ was accepted as the average $\dot{\mathrm{VO}}_{2}$ over the last 30 seconds of the final completed PACER stage. The authors recognize that $20 \mathrm{mSRT}$ performance scores are influenced by a variety of factors other than aerobic fitness, and they note that body mass impacts on estimated peak $\dot{\mathrm{VO}}_{2}$ and that predicted peak $\dot{\mathrm{VO}}_{2}$ is significantly underestimated in individuals with a high body mass index. They resolve that including a variable related to body composition in their prediction equation is critical, and the equation developed includes total number of laps completed during the PACER, body mass index, and the interaction between sex and age. Scott et al (47) conclude that their prediction equation can be used with confidence to estimate 10- to 15-yearolds' $\mathrm{VO}_{2}$ in the final stage of the PACER test.

Weston et al (59) investigated the physical fitness of 9-year-old children from a socially deprived area in North East England. They report data from a battery of physical fitness tests with aerobic fitness "indirectly assessed via 20mSRT performance using the British National Coaching Foundation protocol." The authors present their 20mSRT performance scores not as estimated peak $\dot{\mathrm{VO}}_{2}$ but as number of completed shuttles. In accord with the extant literature, they noted that body mass index was a strong predictor of $20 \mathrm{mSRT}$ performance, that children with a higher body mass ran fewer 20-m shuttles, and that there was a large gender difference in $20 \mathrm{mSRT}$ performance. Boys, on average, completed $37 \%$ more shuttles than girls which is intriguing as, in 9-year-olds, the mean sex difference in aerobic fitness is $\sim 10 \%$, the sex difference in mean sprint speed is $\sim 3 \%$, and, at the time of writing, the world's best recorded sprint performance by a 9-year-old (boy or girl) is by a girl (3). Why there is such a large gender difference in 20mSRT performance scores and not similar sex differences in either aerobic fitness or running speed warrants further investigation. Weston et al (59) reported their 20mSRT performance scores to be not substantially different from those recorded in other regions of England, but the authors found scores from other fitness tests in the battery to be lower than those of children from elsewhere. Overall, they interpret their data to indicate suboptimal levels of physical fitness and to support the introduction of longitudinal physical fitness testing programs within the school setting - a topic that has proved controversial for decades (eg, 17,18,49).

Welsman (55) emphasizes that scientific rigor and critical analysis are essential components of pediatric research and challenges the validity and reliability of predictions of peak $\mathrm{VO}_{2}$ from field performance tests. She describes some of the interpretations of field test scores that have clouded understanding of children's aerobic fitness, its development, and its relationship with current and future health. She asks why, when there is unequivocal evidence that aerobic fitness varies with age and maturity status-driven changes in a range of morphological and physiological covariates and not in direct ratio with body mass, performance test predictions of age-specific peak $\dot{\mathrm{VO}}_{2}$ divided by body mass have been collated into international norms and recommendations for health surveillance. Her most serious concern in relation to child health and well-being is the promotion of performance test scores to establish "clinical red flags" designed to identify individual children and adolescents at risk of cardiovascular disease. She appeals to pediatric exercise scientists that, "our children deserve better than this."

\section{Peak Oxygen Uptake and Cardiovascular Health}

The pediatric origins of atherosclerosis were first reported at the beginning of the 20th century $(45)$, but it was the 1970s $(29,60)$ before aerobic fitness was investigated in relation to cardiovascular risk factors in children. Since then there has been a plethora of publications devoted to the association of both directly determined and predicted peak $\dot{\mathrm{VO}}_{2}$ in childhood and adolescence with present and future cardiovascular risk (28). The topic is currently an issue of lively debate with the validity of field performance test predictions of peak $\dot{\mathrm{VO}}_{2}$ and the impact of peak $\dot{\mathrm{VO}}_{2}$ ratio scaled with body mass on purported associations between youth aerobic fitness and cardiovascular risk factors being challenged (eg, 8,37,51).

Agbaje et al (1) contribute to the ongoing discussion by investigating, in 329 children (aged 8-11 y), the association of arterial stiffness and arterial dilatation in response to a bout of exercise with peak $\dot{\mathrm{VO}}_{2}$ and body fat percentage. The authors assessed the stiffness index (measure of arterial stiffness) and the reflection index (measure of arterial dilatation capacity) using pulse contour analysis before and after a maximal exercise test, body fat content and lean body mass were measured using bioelectrical impedance, and peak $\mathrm{VO}_{2}$ was determined using cycle ergometry and respiratory gas analysis. Neither peak $\mathrm{VO}_{2}$ nor body fat percentage was associated with arterial stiffness in either girls or boys. Decreased body fat percentage and increased peak $\dot{\mathrm{VO}}_{2}$ in ratio with lean body mass were, however, independently associated 
with increased arterial dilatation capacity in response to a bout of exercise in boys but not in girls. The authors discuss plausible explanations for the sex difference but clearly more research is required. Agbaje et al (1) conclude that increasing aerobic fitness and decreasing adiposity may improve arterial health, particularly in boys, and may be important in reducing cardiovascular risk in adulthood.

\section{Peak Oxygen Uptake and High-Intensity Interval Training}

The practice of high-intensity interval training (HIIT) with adults was developed through the first half of the 20th century by Scandinavian athletes before being popularized by Emil Zatopeck following the second world war and featuring regularly in the scientific literature in the 1950s (3). It is, however, only recently that a concerted effort has focused on refining HIIT programs as a means of enhancing young people's aerobic fitness (52).

Baquet et al (12) have been at the forefront of HIIT research, and in their current article, they report the effects of recovery mode on peak $\mathrm{V}_{2}$, time to exhaustion, and time spent at high percentages of peak $\mathrm{VO}_{2}$ during short, high-intensity intermittent runs. Peak $\mathrm{VO}_{2}$ was determined using respiratory gas analysis during a graded running test on a coned $150-\mathrm{m}$ track, and the speed in the last completed stage was considered as the maximal aerobic speed. In 2 subsequent randomized experimental sessions, the children performed short, high-intensity exercise bouts consisting of repeated 15-second runs at $120 \%$ of maximal aerobic speed until exhaustion. In 1 session, the exercise bouts were interspersed with 15 -second active recovery periods set at $50 \%$ of maximal aerobic speed and in the other with 15-second periods of passive recovery. Peak $\mathrm{VO}_{2}$ achieved and time spent above $80 \%$ and $90 \%$ of peak $\mathrm{VO}_{2}$ were not different with active or passive recovery modes, whereas the time to reach peak $\mathrm{VO}_{2}$ was faster with active recovery periods and the time to exhaustion was longer with passive recovery periods. On the basis of their results, the authors propose that either mode of recovery can be used in programs designed to improve children's aerobic fitness with the choice of recovery mode dependent on the overall objectives of the training program.

\section{Cardiopulmonary Exercise Testing}

Dan Cooper has been an inspirational figure in pediatric exercise medicine for over 30 years and his forward looking review focuses on new pathways necessary to advance pediatric exercise medicine and health. In an invited contribution which nicely complements those of van Brussel et al (54) and McNarry (35), Cooper (19) argues that a major barrier to more accurate and effective clinical use of CPET in children is that data analytics and testing protocols have failed to keep pace with enabling techniques and computing capacities. Controversies surrounding the determination and verification of maximal values of $\mathrm{VO}_{2}$ in CPETs are addressed, and the wealth of submaximal data that can be collected during a CPET is highlighted. He discusses the power of submaximal slope analysis, outlines the progress of gas analysis techniques, and shows how the emergence of breath-by-breath analysis has opened up new avenues of research. Novel but sparse research in respiratory gas kinetics resulting in the enrichment of CPET data from both sick and healthy children is analyzed, and how the development of advanced technologies and enhanced computing capacity have enabled CPET data analysis using biological variability and machine learning is reviewed. Cooper indicates that although early research has been promising, further work is required to exploit the opportunities offered by these techniques. He suggests that the key challenge facing pediatric exercise scientists and clinicians is the development of testing strategies that can be used to gauge aerobic fitness robustly, repeatedly, and cost effectively. He concludes his contribution with a list of recommendations that have emerged from an international working group established to identify the challenges facing optimal utilization of exercise testing in child health research.

Van Brussel et al (54) draw on 15 years' experience of working in one of Europe's leading pediatric exercise laboratories to describe an innovative 7-step systematic approach to interpreting CPET data for diagnostic, prognostic, and evaluative purposes. The authors address methodological and physiological challenges in CPETs with pediatric clinical populations. Notably, they not only discuss the assessment and interpretation of peak $\dot{\mathrm{VO}}_{2}$ but also recognize that abnormal CPET responses can be caused by multiple physiological systems. Cardiovascular, respiratory, gas exchange, and muscle metabolic responses to submaximal and maximal exercise are reviewed. The clinical importance of understanding deconditioning in exercise-induced physiological responses during a CPET is explained, and the relevance of the child's symptom perception is acknowledged. The final step in their systematic approach stresses the importance of a comprehensive and standardized CPET report including a clear interpretation of the exercise data. The Commentary is enhanced with a series of clear and informative tables and figures, which share the comprehensive experience of the laboratory team.

\section{Pulmonary Oxygen Uptake Kinetics}

Macek and Vavra (32) were probably the first to report the halftime of children's transient responses at the onset of exercise, but the initial application of breath-by-breath technology to youth pulmonary $\mathrm{VO}_{2}$ kinetics was by Cooper et al (21). Fawkner et al (25-27) were the first to demonstrate that, in conflict with data from adults, there is no significant relationship in children between peak $\dot{\mathrm{VO}}_{2}$ and pulmonary $\dot{\mathrm{VO}}_{2}$ kinetics at the onset of either moderate- or heavy-intensity exercise. Unique insights into the development of both aerobic fitness (13) and muscle exercise metabolism (4) rest in the pulmonary $\mathrm{VO}_{2}$ response to the onset of exercise in well-defined intensity domains but, despite the advanced technologies and enhanced computing capacity outlined herein by Cooper (19), rigorous investigations of respiratory gas kinetics in childhood and adolescence are sparse-reflected by the observation that despite advertising an invited review, there were no original research submissions on pulmonary $\mathrm{VO}_{2}$ kinetics in the Call for Papers for this Special Issue.

Mel McNarry (35) focuses her invited review on pulmonary $\mathrm{VO}_{2}$ kinetics in youth and emphasizes the need for rigor in the analysis of data with a low signal to noise ratio. She discusses how flawed methodology and misinterpretation of rich data sets have clouded understanding of young people's transient responses at the onset and offset of exercise. She discusses the theory underpinning pulmonary $\mathrm{VO}_{2}$ response and recovery profiles, comments on their known and potential clinical applications, and discusses the few secure data on the influence of age and sex on youth $\mathrm{VO}_{2}$ kinetics. She points out that only 2 longitudinal studies have been published, and there are no data on the effects of maturity status on pulmonary $\dot{\mathrm{VO}}_{2}$ kinetics. Much of what we know of the trainability 
of young people's $\dot{\mathrm{V}} \mathrm{O}_{2}$ kinetics is founded on McNarry's own research. She outlines the principal findings but stresses that they are predominately reliant on cross-sectional comparisons of trained and untrained youth athletes with only 1 intervention study (with obese children) in the extant literature. One of the strengths of the review is a discussion of the potential mechanistic bases of age-, sex-, and training-induced changes in pulmonary $\mathrm{VO}_{2}$ kinetics. McNarry (35) concludes that more methodologically rigorous studies are required to resolve the influence of and interactions between sex, age, maturity status, and external stimuli (eg, training) on the development of the dynamic pulmonary $\dot{\mathrm{VO}}_{2}$ responses at the onset and offset of exercise.

In a Letter to the Editor, Dotan (22) argues that children are more limited than adults in their capacity to recruit and utilize higher threshold type II motor units and reviews the role of the child-adult differential muscle-activation hypothesis in explaining why children have faster $\mathrm{V}_{2}$ kinetics than adults. $\mathrm{He}$ suggests that the hypothesis is able to account, as a sole or major underlying factor, for all the mechanisms, proposed by McNarry (35), as partial explanations for adult-child differences. In her response, McNarry (34) acknowledges that different child-adult fiber-type distribution and recruitment may contribute to an exploration of child-adult differences but contends that there is insufficient evidence to support the view that the differential muscle-activation hypothesis provides the sole or major explanation. She takes the opportunity to expand her analysis of the sparse research, critically examines the evidence supporting the existence of child-adult differences, questions aspects of the differential muscle-activation hypothesis, and identifies areas for further research. Taken together, the thought-provoking contributions of Dotan $(22)$ and McNarry $(34,35)$ remind us that much remains to be learned about the mechanisms underpinning the development of pulmonary $\dot{\mathrm{V}}_{2}$ dynamics in childhood and adolescence.

\section{Future Directions}

It is readily apparent from contributions to this Special Issue that, despite 80 years of intensive investigation of young people's peak $\dot{\mathrm{VO}}_{2}$ and its relationship with current and future health, controversies persist. Recent research using sophisticated mathematical modeling techniques and evolving technologies has begun to elucidate the development of pulmonary $\mathrm{V}_{2}$ kinetics but rigorously determined data are sparse. A major research challenge is to explain the mechanisms underlying peak $\mathrm{V}_{2}$ and pulmonary $\dot{\mathrm{V}}_{2}$ kinetics and how they develop in childhood and adolescence. The reviews, commentaries, research papers, and letters in this Special Issue of Pediatric Exercise Science address several of the most contentious issues, present new data, scrutinize the evidence base, and express opinions that will hopefully generate further discussion in Pediatric Exercise Science and pediatrics generally. Collectively, the articles emphasize the fundamental requirement for scientific and statistical rigor when assessing and interpreting youth aerobic fitness and invite future studies to

1. incorporate advanced technologies, innovative statistical modeling, and increased computing capacity into CPETs and rigorously analyze integrated submaximal, maximal, and kinetic responses from multiple physiological systems;

2. interpret aerobic fitness in relation to sex-specific concurrent changes in age- and maturity status-driven morphological and physiological variables;
3. elucidate the mechanisms underpinning sexual dimorphism and age- and maturity status-driven changes in aerobic fitness during growth and maturation;

4. verify and evaluate purported relationships between aerobic fitness and indicators of health and well-being; and

5. ensure that aerobic fitness recommendations designed to promote young people's present and future health and well-being are based on sound scientific evidence.

\section{References}

1. Agbaje AO, Haapala EA, Lintu N, et al. Associations of cardiorespiratory fitness and adiposity with arterial stiffness and arterial dilatation capacity in response to a bout of exercise in children. Pediatr Exerc Sci. 2019;31(2). doi:10.1123/pes.2018-0145

2. Armstrong N. Commentary on the assessment and interpretation of pediatric aerobic fitness-the year that was 2017. Pediatr Exerc Sci. 2018;30:12-18. PubMed ID: 29424279 doi:10.1123/pes.2017-0287

3. Armstrong N. Development of the Youth Athlete. Oxford, UK: Routledge; 2018. pp. 97-100, 163-173.

4. Armstrong N, Barker AR, McManus AM. Muscle metabolism during exercise. In: Armstrong N, van Mechelen W, editors. Oxford Textbook of Children's Sport and Exercise Medicine. 3rd ed. Oxford, UK: Oxford University Press; 2017. pp. 69-87.

5. Armstrong N, Welsman J. Development of aerobic fitness during childhood and adolescence. Pediatr Exerc Sci. 2000;12:128-149. doi:10.1123/pes.12.2.128

6. Armstrong N, Welsman J. Development of peak oxygen uptake from 11-16 years determined using both treadmill and cycle ergometry. Eur J Appl Physiol. 2019;119:801-812. PubMed ID: 30627827 doi:10.1007/s00421-019-04071-3

7. Armstrong N, Welsman J. Sex-specific longitudinal modeling of youth peak oxygen uptake. Pediatr Exerc Sci. 2019;31(2). doi:10. 1123/pes.2018-0175

8. Armstrong N, Welsman J. Twenty-metre shuttle run: (mis)representation, (mis)interpretation and (mis)use. Br J Sports Med. Epub ahead of print. doi:10.1136/bjsports-2018-100082

9. Armstrong N, Welsman JR, Kirby BJ. Peak oxygen uptake and maturation in 12-year-olds. Med Sci Sports Exerc. 1998;30:165-169. PubMed ID: 9475659 doi:10.1097/00005768-199801000-00023

10. Åstrand PO. Experimental Studies of Physical Working Capacity in Relation to Sex and Age. Copenhagen, Denmark: Munksgaard; 1952.

11. Azedo P, Bhammar DM, Babb TG, et al. Commentaries on Viewpoint: $\mathrm{V}_{2}$ peak is an acceptable estimate of cardiorespiratory fitness but not $\dot{\mathrm{VO}_{2}}$ max. J Appl Physiol. 2018;125:233-240. doi:10.1152/ japplphysiol.00319.2018

12. Baquet G, Dupont G, Gamelin FX, Autocourier J, Berthoin S. Active versus passive recovery in high-intensity intermittent exercises in children: An exploratory study. Pediatr Exerc Sci. 2019;31(2). doi:10.1123/pes.2018-0218

13. Barker AR, Armstrong N. Pulmonary oxygen uptake kinetics. In: Armstrong N, van Mechelen W, editors. Oxford Textbook of Children's Sport and Exercise Medicine. 3rd ed. Oxford, UK: Oxford University Press; 2017. pp. 181-194.

14. Barker AR, Williams CA, Jones AM, Armstrong N. Establishing maximal oxygen uptake in young people during a ramp test to exhaustion. Brit J Sports Med. 2011;45:498-503. doi:10.1136/ bjsm.2009.063180

15. Bar-Or O, Rowland TW. Pediatric Exercise Medicine. Champaign, IL: Human Kinetics; 2004. pp. 5-7. 
16. Blais S, Blanchard J, Dallaire F. An alternative to ratio scaling for the interpretation of aerobic fitness in children. Pediatr Exerc Sci. 2019;31(2). doi:10.1123/pes.2019-0008

17. Cale L, Harris J. Fitness testing in physical education-a misdirected effort in promoting health, lifestyle and physical activity. Phys Educ Sport Pedagogy. 2009;14:89-108. doi:10.1080/17408980701345782

18. Cohen DD, Voss C, Sandercock GRH. Fitness testing for children: Let's mount the zebra! J Phys Act Health. 2015;12:597-603. PubMed ID: 24905807 doi:10.1123/jpah.2013-0345

19. Cooper DM. Exercise science and child health: a tale of many journeys. Pediatr Exerc Sci. 2019;31(2). doi:10.1123/pes.2018-0259

20. Cooper DM. Rethinking $\mathrm{V}_{2}$ max: right problem, wrong solution (Letter to the Editor regarding Poole and Jones' 'Measurement of maximum oxygen uptake $\dot{\mathrm{V}} \mathrm{O}_{2}$ max: $\dot{\mathrm{VO}}_{2}$ peak is no longer acceptable'). J Appl Physiol. 2017;123:498. PubMed ID: 28830932 doi:10. 1152/japplphysiol.00396.2017

21. Cooper DM, Berry C, Lamarra N, Wassermann K. Kinetics of oxygen uptake and heart rate at onset of exercise in children. $J$ Appl Physiol. 1985;59:211-217. PubMed ID: 4030564 doi:10.1152/jappl.1985.59. 1.211

22. Dotan R. Why do children have faster $\mathrm{V}_{2}$ kinetics? Pediatr Exerc Sci. 2019;31(2). doi:10.1123/pes.2019-0015

23. Eliakim A, Falk B, Armstrong N, et al. Expert's choice: 2018's most exciting research in the field of pediatric exercise science. Pediatr Exerc Sci. 2019;31:1-27. PubMed ID: 30760123 doi:10.1123/pes. 2019-0010

24. Falk B, Dotan R. Measurement and interpretation of maximal aerobic power in children. Pediatr Exerc Sci. 2019;31(2). doi:10.1123/pes. 2018-0191

25. Fawkner SG, Armstrong N. Longitudinal changes in the kinetic response to heavy-intensity exercise in children. J Appl Physiol. 2004;97:460 466. PubMed ID: 15033964 doi:10.1152/japplphysiol.00784.2003

26. Fawkner SG, Armstrong N. Sex differences in the oxygen uptake kinetic response to heavy-intensity exercise in prepubertal children. Eur J Appl Physiol. 2004;93:210-216. PubMed ID: 15309549 doi:10.1007/s00421-004-1201-7

27. Fawkner SG, Armstrong N, Potter CR, Welsman JR. Oxygen uptake kinetics in children and adults after the onset of moderate intensity exercise. J Sports Sci. 2002;20:319-326. PubMed ID: 12003277 doi:10.1080/026404102753576099

28. Ferreira I, Twisk JWR. Physical activity, cardiorespiratory fitness, and cardiovascular health. In: Armstrong N, van Mechelen W, editors. Oxford Textbook of Children's Sport and Exercise Medicine. 3rd ed. Oxford, UK: Oxford University Press; 2017. pp. 239-253.

29. Gilliam TB, Katch VL, Thorland W, Weltman A. Prevalence of coronary heart disease risk factors in active children, 7 to 12 years of age. Med Sci Sports. 1977;9:21-25. PubMed ID: 859401

30. Hale T. History of developments in sport and exercise physiology: A.V. Hill, maximal oxygen uptake, and oxygen debt. J Sports Sci. 2008;26:365-400. PubMed ID: 18228167 doi:10.1080/02640410 701701016

31. Loftin M, Sothern M, Abe T, Bonis M. Expression of $\dot{V}_{2}$ peak in children and youth with special reference to allometric scaling. Sports Med. 2016;46:1451-1460. PubMed ID: 27139725 doi:10.1007/ s40279-016-0536-7

32. Macek M, Vavra J. The adjustment of oxygen uptake at the onset of exercise: a comparison between pre-pubertal boys and young adults. In J Sports Med. 1980;1:70-72.

33. Mayorga-Vega D, Aguiler-Soto P, Viciana J. Criterion-related validity of the 20-m shuttle run test for estimating cardiorespiratory fitness: a meta-analysis. J Sports Sci Med. 2015;14:536-547. PubMed ID: 26336340
34. McNarry MA. Why do children have faster $\dot{\mathrm{VO}}_{2}$ kinetics? - a response to Dotan (2019) Pediatr Exerc Sci. 2019;31(2). doi:10.1123/pes.20190032

35. McNarry MA. Oxygen uptake kinetics in youth: characteristics, interpretation, and application. Pediatr Exerc Sci. 2019;31(2). doi:10. 1123/pes.2018-0177

36. Mintjens S, Menting MD, Daams JG, van Poppel MNM, Roseboom TJ, Gemke RJBJ. Cardiorespiratory fitness in childhood and adolescence affects future cardiovascular risk factors: a systematic review of longitudinal studies. Sports Med. 2018;48:2577-2605. PubMed ID: 30144022 doi:10.1007/s40279-018-0974-5

37. Mintjens S, Menting MD, Daams JG, van Poppel MNM, Roseboom TJ, Gemke RJBJ. Reply to Tarp et al.: Comment on: 'Cardiorespiratory fitness in childhood and adolescence affects future cardiovascular risk factors: a systematic review of longitudinal studies'. Sports Med. 2019;49:163-165. PubMed ID: 30593651 doi:10.1007/s40279018-01042-0

38. Pate RR. The case for large-scale physical fitness testing in American youth. Pediatr Exerc Sci. 1989;1:290-294. doi:10.1123/ pes.1.4.290

39. Poole DC, Jones AM. Measurement of maximum oxygen uptake $\dot{\mathrm{V}} \mathrm{O}_{2} \mathrm{max}$ : $\dot{\mathrm{V}} \mathrm{O}_{2}$ peak is no longer acceptable. J Appl Physiol. 2017; 122:997-1002. PubMed ID: 28153947 doi:10.1152/japplphysiol. 01063.2016

40. Poole DC, Jones AM. Reply to Cooper's letter in reference to: measurement of maximum oxygen uptake $\dot{\mathrm{VO}}_{2}$ max: $\dot{\mathrm{VO}}_{2}$ peak is no longer acceptable. J Appl Physiol. 2017;123:499. PubMed ID: 28830933 doi:10.1152/japplphysiol.00442.2017

41. Robinson S. Experimental studies of physical fitness in relation to age. Arbeitsphysiologie. 1938;10:251-323.

42. Rowland TW. Fitness testing in children. Where from here? Pediatr Exerc Sci. 1989;1:289. doi:10.1123/pes.1.4.289

43. Rowland TW. The horse is dead; let's dismount. Pediatr Exerc Sci. 1995;7:117-120. doi:10.1123/pes.7.2.117

44. Ruiz JR, Cavero-Redondo I, Ortega FB, Welk GJ, Andersen LB, Martinez-Vizcaino V. Cardiorespiratory fitness cut points to avoid cardiovascular disease risk in children and adolescents; what level of fitness should raise a red flag? A systematic review and meta-analysis. Br J Sports Med. 2016; 50:1451-1458. PubMed ID: 27670254 doi:10.1136/bjsports-2015-095903

45. Saltykov S. Jugenoliche ubd beginnende atheroscklerse. Korrespondezblatt fur Schweizer Aertz Basel. 1915;1057. Cited by: Voller RD, Strong WB. Pediatric aspects of atherosclerosis. Am Heart J. 1981; 101:815-23.

46. Sansum KM, Weston ME, Bond B, et al. Validity of the supramaximal test to verify maximal oxygen uptake in children and adolescents. Pediatr Exerc Sci. 2019;31(2). doi:10.1123/pes.2018-0129

47. Scott SN, Springer CM, Oody JF, McClanahan MS, Wiseman BD, Kybartas TJ, Coe DP. Development and validation of a PACER prediction equation for $\mathrm{V}_{2}$ peak in 10- to 15- year-old youth. Pediatr Exerc Sci. 2019;31(2). doi:10.1123/pes.2018-0136

48. Seefeldt V, Vogel P. Physical fitness testing of children: a 30 year history of misguided efforts. Pediatr Exerc Sci. 1989;1:295-302. doi:10.1123/pes.1.4.295

49. Shepard RJ. Physical Activity and Growth. Chicago, IL: Year Book Medical Publishers; 1982:60-73.

50. Tanner JM. Fallacy of per-weight and per-surface area standards and their relation to spurious correlation. J Appl Physiol. 1949;2:1-15. PubMed ID: 18133122 doi:10.1152/jappl.1949.2.1.1

51. Tarp J, Dalene KE, Steene-Johannessen J, Ekelund U. Comment on: 'Cardiorespiratory fitness in childhood and adolescence affects future cardiovascular risk factors: a systematic review of longitudinal 
studies'. Sports Med. 2019;49:159-161. PubMed ID: 30593650 doi:10.1007/s40279-018-01035-z

52. Tolfrey K, Smallwood J. High-intensity interval training. In: Armstrong N, van Mechelen W, editors. Oxford Textbook of Children's Sport and Exercise Medicine. 3rd ed. Oxford, UK: Oxford University Press; 2017:477-491.

53. Tomkinson GR, Lang JJ, Blanchard J, Leger L, Tremblay MS. The 20-m shuttle run: assessment and interpretation of data in relation to youth aerobic fitness and health. Pediatr Exerc Sci. 2019;31(2). doi:10.1123/pes.2018-0179

54. van Brussel M, Bongers BC, Hulzebos EHJ, Burghard M, Takken T. A systematic approach to interpreting the cardiopulmonary exercise test in pediatrics. Pediatr Exerc Sci. 2019;31(2). doi:10.1123/pes. 2018-0235

55. Welsman J. Reflecting on field performance tests of pediatric aerobic fitness: after 30 years it really is time to move on. Pediatr Exerc Sci. 2019;31(2). doi:10.1123/pes.2019-0034
56. Welsman J, Armstrong N. Interpreting aerobic fitness in youth: alternatives to ratio scaling - a response to Blais et al (2019). Pediatr Exerc Sci. 2019;31(2). doi:10.1123/pes.2019-0026

57. Welsman J, Armstrong N. Interpreting aerobic fitness in youth: the fallacy of ratio scaling. Pediatr Exerc Sci. 2019;31(2). doi:10.1123/ pes.2018-0141

58. Welsman JR, Armstrong N, Kirby BJ, Nevill AM, Winter EM. Scaling peak $\dot{\mathrm{VO}}_{2}$ for differences in body size. Med Sci Sports Exerc. 1996;28:259-265. PubMed ID: 8775163 doi:10.1097/00005768199602000-00016

59. Weston KA, Pasencinic N, Basterfield L. A preliminary study of physical fitness in 8- to 10-year-old primary school children from North East England in comparison to national and international data. Pediatr Exerc Sci. 2019;31(2) doi:10.1123/pes.2018-0135

60. Wilmore JH, McNamara JJ. Prevalence of coronary heart disease risk factors in boys, 8 to 12 years of age. J Pediatr. 1974;84:527-533. PubMed ID: 4834246 doi:10.1016/S0022-3476(74)80672-4 